\title{
Water Exchange Versus Split-Dose Bowel Preparation and the ADR: Is WE There Yet?
}

\author{
Felix W. Leung ${ }^{1,2} \cdot$ Hui Jia ${ }^{1,2,3}$
}

Published online: 15 February 2018

(c) This is a U.S. Government work and not under copyright protection in the US; foreign copyright protection may apply 2018

The adenoma detection rate (ADR), which is defined as the percentage of initial screening colonoscopies in averagerisk subjects $>50$ years in which one or more adenomatous polyps was identified, has become a de facto measure of procedural quality. Although affected by factors beyond the endoscopist's control such as the quality of bowel preparation, data strongly support an inverse correlation between the incidence of interval cancers and a low ADR [1]. The adverse effects of bowel preparation on the ADR have encouraged considerable research into improvement in bowel preparation quality. Inadequate bowel preparation decreased, whereas high-quality cleansing increased the ADR, particularly for flat lesions (e.g., sessile serrated polyps) [2]. Screening colonoscopy is not a cost-effective strategy when compared with fecal immunochemical test if the inadequate bowel preparation rate is $>13 \%$ [3].

In this issue of Digestive Diseases and Sciences, Wang et al. [4] describe the effect of changing from a single- to a split-dose preparation on colonoscopy quality measures in a retrospective cohort study of quality indicators that relied on electronic medical record data. Cases were identified via the International Classification of Diseases (ICD)-9 code V76.51, "special screening for malignant neoplasms of colon." Single-dose preparation data were collected from September 1, 2013, to August 31, 2014. The split-dose

Felix W. Leung

felix.leung@va.gov

Hui Jia

jiahuifmmu@163.com

1 Gastroenterology, Medicine, Sepulveda Ambulatory Care Center, Veterans Affairs Greater Los Angeles Healthcare System, 111G, 16111 Plummer Street, North Hills, CA 91343, USA

2 David Geffen School of Medicine at UCLA, Los Angeles, CA, USA

3 Xijing Hospital of Digestive Diseases, Fourth Military Medical University, Xi' an, Shaanxi, China preparation was implemented in November 2014, with associated data collected from January 1, 2015, to August 31, 2015. The results included 1602 colonoscopies in the singledose group and 1061 colonoscopies in the split-dose group. The Boston Bowel Preparation Scale (BBPS) score was significantly improved in the split-dose group (8.64 vs. 8.25 , $P<.0001)$. There was no significant difference in ADR (40.7 vs. $40.5 \%, P=0.92$ ). The rate for recommending an early repeat examination due to an inadequate bowel preparation, however, was significantly decreased in the split-dose group ( 3.9 vs. $8.9 \%, P<0.0001)$. The authors concluded that while split-dose preparation significantly improved overall bowel cleanliness, there was no significant improvement in the high baseline ADR, suggesting an ADR threshold over which improved bowel preparation would have little impact. The significantly reduced frequency that inadequate preparation prompted an early repeat examination had important clinical implications on performance, costs, and patient experience, providing further evidence supporting the use of split-dose preparation. Although this report is limited by its retrospective nature, the strengths rest with a fairly large sample size, the data showing patients with mostly excellent BBPS scores before (8.25) and after (8.64) changing to split-dose preparation, and information on the frequency with which an inadequate preparation prompted the recommendation of an early repeat examination.

Early reports of split-dose preparation improving bowel cleanliness did not include ADR data. Although one recent randomized controlled trial (RCT) did report a significantly improved ADR with split-dose bowel preparation [5], there were important barriers to the use of split-dose [6]. In the report describing split-dose increasing ADR as the primary endpoint, 50-69-year-old subjects undergoing first colonoscopy after positive fecal immunochemical test were randomized 1:1 to receive low-volume $2 \mathrm{~L}$ polyethylene glycol (PEG)-ascorbate solution in a "split-dose" (Split-Dose Group, SDG) or a "day-before" regimen (Day-Before Group, DBG) in 690 subjects. At per-patient analysis, ADR was 
significantly higher in the SDG than in the DBG (183/345, $53.0 \%$ vs. $141 / 345,40.9 \%$, relative risk (RR) $1.22,95 \%$ CI 1.03-1.46) [5]. These authors also evaluated the patient preference for the split-dose and day-before regimens and their association with patient-related factors using a 20 -item questionnaire. Of 1447 patients (mean age $59.2 \pm 13.5$ years, men $54.3 \%$ ), 61.7 and $38.3 \%$ chose a split-dose and daybefore regimens, respectively. A linear correlation was observed between the time of colonoscopy appointments and split-dose preference, from $27.3 \%$ in patients scheduled for an 08:00 procedure to $96 \%$ in patients scheduled for 14:00 $\left(P<0.001, \chi^{2}\right.$ for linear trend $)$. Multivariate analysis revealed that a colonoscopy appointment before 10:00, travel time to the endoscopy unit $>1 \mathrm{~h}$, low education level, and female gender were inversely correlated with the preference for the split-dose regimen. Overall, the risk of travel interruption and fecal incontinence was slightly increased in split regimen patients (3.0 vs. $1.4 \%$ and 1.5 vs. $0.9 \%$, respectively; $P=\mathrm{NS}$ ). The authors concluded that patient attitude toward the split-dose regimen is suboptimal, especially for early morning examinations [6].

Depending on the diet before colonoscopy, the type of preparation used, and an examination time scheduled for the morning or afternoon, split-dose preparation produced BBPS scores from 6.5 to 7.9 [7]. How different levels of bowel preparation scores influence ADR is unclear. For example, if a patient has a poor bowel preparation, residual feces could obscure adenomas, especially flat lesions. Conversely, if a patient does not have an adenoma in the first place, good or excellent bowel preparation would have no bearing on the ADR.

The conventional insertion technique with $\mathrm{CO}_{2}$ or air insufflation (AI) calls for expeditious intubation of the cecum with cleaning performed during withdrawal. In the current report, BBPS was high (8.25) even before the adoption of the split-dose regimen. The authors did not mention how much additional cleaning was performed during colonoscopy before bowel cleanliness was scored using the BBPS. They found that the time required for colonoscopy completion was shortened by nearly 2 min with the splitdose preparation. They speculated that the time savings may have been due to a reduced need to suction and wash the bowel. In an earlier retrospective report [2], patients received split-dose regimen of $4 \mathrm{~L}$ polyethylene glycol (PEG-3350) solution for morning colonoscopies or a same-day regimen of 4L PEG-3350 for afternoon examinations. The report [2] discussed intra-procedural bowel cleansing as a solution for patients with inadequate bowel preparation. The practice could reduce the need for early repeat colonoscopy, thereby reducing costs, and patient and endoscopist time and inconvenience while potentially improving outcomes [7].

The water exchange (WE) method was initially developed to facilitate cecal intubation in unsedated veterans in the USA. The need to minimize painful colonic maneuvering such as elongation and distention called for no air insufflation and removal of residual air during insertion, using water to expand the colon. The net effect of using clean water to guide insertion and the removal of soiled water to minimize distention augmented bowel cleaning during insertion while reducing or obviating the need for cleaning during withdrawal. Compared with traditional AI in patients purged with split-dose bowel preparation, the WE method significantly increased BBPS scores and the ADR. Based on the analysis of withdrawal phase videos of WE and AI, reduced endoscopist distraction and more focused withdrawal inspection were identified as possible
Table 1 Comparison of WE and $\mathrm{AI}$ in RCT

\begin{tabular}{|c|c|c|c|c|}
\hline Reference & & WE & AI & $P$ \\
\hline \multirow[t]{5}{*}{ Jia et al. [8] } & Number of subjects & 1653 & 1650 & \\
\hline & Overall BBPS score* & $7.3 \pm 1.6$ & $7.0 \pm 2.3$ & $<0.001$ \\
\hline & Right colon BBPS score* & $2.3 \pm 0.7$ & $2.2 \pm 1.5$ & $<0.014$ \\
\hline & Transverse colon BBPS score* & $2.5 \pm 0.6$ & $2.4 \pm 0.7$ & $<0.001$ \\
\hline & Overall ADR & $18.3 \%$ & $13.4 \%$ & $<0.001$ \\
\hline \multirow[t]{4}{*}{ Hsieh et al. [9] } & Number of subjects & 217 & 217 & \\
\hline & Overall BBPS score* & $7.1 \pm 1.3$ & $6.2 \pm 1.1$ & $<0.001$ \\
\hline & Overall ADR intention-to-treat analysis & $49.8 \%$ & $37.8 \%$ & 0.016 \\
\hline & Overall ADR per-protocol analysis & $51.0 \%$ & $38.1 \%$ & 0.010 \\
\hline \multirow[t]{4}{*}{ Cadoni et al. [10] } & Number of subjects & 408 & 408 & \\
\hline & Overall BBPS score ${ }^{\#}$ & $9.0(7.0-9.0)$ & $8.0(6.0-9.0)$ & $<0.05$ \\
\hline & Right colon BBPS score ${ }^{\#}$ & $3.0(2.0-3.0)$ & $2.0(2.0-3.0)$ & $<0.03$ \\
\hline & Overall ADR & $49.3 \%$ & $40.4 \%$ & 0.03 \\
\hline
\end{tabular}

*Mean $\pm \mathrm{SD} ;{ }^{*}$ Median (IQR)

$B B P S$ Boston Bowel Preparation Scale, $W E$ water exchange, $A I$ air insufflation

$P$ values are based on analyses using relative risk or $\chi^{2}$ 
advantages of the WE as compared with the AI insertion methods.

In three recent RCT involving subjects undergoing $\mathrm{AI}$ and WE colonoscopy, insertion cleansing is an integral component of WE colonoscopy [8-10]. The WE method significantly improved bowel cleanliness after patients received split-dose preparation and increased ADR (Table 1). In the RCT completed in mainland China [8], the WE method significantly improved overall and segmental (right and transverse) colon cleanliness and significantly increased overall ADR (18.3 vs. $13.4 \%, P<0.001)$ [8]. In the RCT completed in Taiwan [9], the BBPS score was $7.1 \pm 1.3$ in the WE group and $6.2 \pm 1.1$ in the AI group, $P<0.001$ [9]. Intention-to-treat and per-protocol analysis indicated that ADR in the WE group was significantly higher than that in the AI group. In order to minimize bias, a third study completed in Europe used different colonoscopists to perform insertion and withdrawal [10]. This European study also confirmed the results of the reports from mainland China and Taiwan. Thus, in patients with adequate $(\geq 6)$ but not necessarily excellent $(>8)$ BBPS scores, the WE method appears to be superior to the AI method in improving bowel preparation quality and ADR. These RCT data support the hypothesis that salvage cleaning by WE during colonoscope insertion is effective in enhancing bowel cleanliness and ADR during withdrawal, even after use of split-dose preparation.

Insertion cleaning of WE reproducibly increased ADR. Prevention of interval cancers by insertion cleaning of WE remains to be demonstrated. A future study in split-dose prepared patients, focused on the impact of WE insertion cleansing compared with traditional AI withdrawal cleaning, on the occurrence of interval cancers deserves to be considered.

Acknowledgments Supported in part by VA Medical research funds.

\section{Compliance with ethical standards}

Conflict of interest The authors have no conflict of interest pertinent to this report to declare.

\section{References}

1. Chaptini L, Laine L. Can I improve my adenoma detection rate? J Clin Gastroenterol. 2015;49:270-281.

2. Oh CH, Lee CK, Kim JW, et al. Suboptimal bowel preparation significantly impairs colonoscopic detection of nonpolypoid colorectal neoplasms. Dig Dis Sci. 2015;60:2294-2303. https://doi. org/10.1007/s10620-015-3628-6.

3. Kingsley J, Karanth S, Revere FL, et al. Cost effectiveness of screening colonoscopy depends on adequate bowel preparation rates-a modeling study. PLOS ONE. 2016;11:e0167452.

4. Wang L, Sprung BS, DeCross AJ, et al. Split-dose bowel preparation reduces the need for early repeat colonoscopy without improving adenoma detection rate. Dig Dis Sci. https://doi. org/10.1007/s10620-017-4877-3.

5. Radaelli F, Paggi S, Hassan C, et al. Split-dose preparation for colonoscopy increases adenoma detection rate: a randomised controlled trial in an organised screening programme. Gut. 2017;66:270-277.

6. Radaelli F, Paggi S, Repici A, et al. Barriers against split-dose bowel preparation for colonoscopy. Gut. 2017;66:1428-1433.

7. Albugeaey M, Audi A, Loughney TM, et al. Superiority of splitdose bowel preparations for morning and afternoon colonoscopies: a single center experience. Gastroenterol Hepatol Intern J. 2017;2:000120.

8. Jia H, Pan Y, Guo X, et al. Water exchange method significantly improves adenoma detection rate: a multicenter, randomized controlled trial. Am J Gastroenterol. 2017;112:568-576.

9. Hsieh $\mathrm{YH}$, Tseng CW, Hu CT, et al. Prospective multicenter randomized controlled trial demonstrating water exchange (WE), but not water immersion (WI), significantly increases adenoma detection compared with air insufflation (AI) even in propofol sedated patients. GIE. 2017;86:192-201.

10. Cadoni S, Falt P, Rondonotti E, et al. Water exchange for screening colonoscopy increases adenoma detection rate: a multicenter, double-blinded, randomized controlled trial. Endoscopy. 2017;49:456-467. 